\title{
Optimization of X-ray absorbers for TES microcalorimeters
}

\author{
Naoko Iyomoto, John E. Sadleir, Enectali Figueroa-Feliciano, Tarek Saab, Simon Bandler, \\ Caroline Kilbourne, James Chervenak, Dorothy Talley, Fred Finkbeiner, Regis Brekosky, \\ Mark Lindeman, Richard Kelley, Frederocl. S. Porter, Kevin Boyce, \\ NASA/GSFC, Greenbelt Rd. Greenbelt, MD 20771, USA
}

\begin{abstract}
We have investigated the thermal, electrical, and structural properties of $\mathrm{Bi}$ and $\mathrm{BiCu}$ films that are being developed as X-ray absorbers for transition-edge sensor (TES) microcalorimeter arrays for imaging X-ray spectroscopy. Bi could be an ideal material for an X-ray absorber due to its high X-ray stopping power and low heat capacity, but it has a low thermal conductivity, which can result in position dependence of the pulses in the absorber. In order to improve the thermal conductivity, we added $\mathrm{Cu}$ layers in between the $\mathrm{Bi}$ layers. We measured electrical and thermal conductivities of the films around $0.1 \mathrm{~K}$, the operating temperature of the TES calorimeter, to examine the films and to determine the optimal thickness of the Cu layer. From the electrical conductivity measurements, we found that the $\mathrm{Cu}$ is more resistive on the $\mathrm{Bi}$ than on a $\mathrm{Si}$ substrate. Together with an SEM picture of the Bi surface, we concluded that the rough surface of the Bi film makes the $\mathrm{Cu}$ layer resistive when the $\mathrm{Cu}$ layer is not thick enough to fill in the roughness. From the thermal conductivity measurements, we determined the thermal diffusion constant to be $2 \times 10^{3} \mu \mathrm{m}^{2} \mu \mathrm{s}^{-1}$ in a film that consists of $2.25 \mu \mathrm{m}$ of $\mathrm{Bi}$ and $0.1 \mu \mathrm{m}$ of $\mathrm{Cu}$. We measured the position dependence in the film and found that its thermal diffusion constant is too low to get good energy resolution, because of the resistive $\mathrm{Cu}$ layer and/or possibly a very high heat capacity of our $\mathrm{Bi}$ films. We show plans to improve the thermal diffusion constant in our $\mathrm{BiCu}$ absorbers.
\end{abstract}

Keywords: TES, microcalorimeter, X-ray, bismuth, thermalization

\section{INTRODUCTION}

We are developing a microcalorimeter array for imaging X-ray spectroscopy for future X-ray astrophysics missions (Ref. 1). The array has $8 \times 8$ pixels. The size of each pixel is $250 \mu \mathrm{m} \times 250 \mu \mathrm{m}$ (Figure 1a). Each pixel consists of a Mo and Au bilayer transition-edge sensor (TES) as a thermometer, a silicon-nitride membrane as a thermal link, and a Bi and $\mathrm{Cu}$ multilayer as an X-ray absorber. We need a close-packed array, so we need the absorbers to touch the TES and extend over the space between pixels in a overhanging structure (Figure 1b and c).

We make our $\mathrm{X}$-ray absorbers out of $\mathrm{Bi}$ because $\mathrm{Bi}$, a semi-metal, has the following advantages over metals. $\mathrm{Bi}$ has a high X-ray stopping power. Bi has a low heat capacity: in general we need the low heat capacity in order to get good energy resolution. Bi does not change the electrical resistance or superconducting transition of the TES even when the $\mathrm{Bi}$ is directly deposited on the TES. In addition, $\mathrm{Bi}$ is brittle, so that it is easy to fabricate the overhanging structure with $\mathrm{Bi}$ in a micro-machining liftoff process.

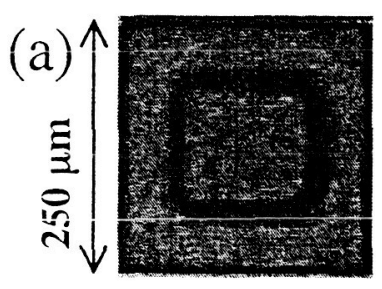

(b)

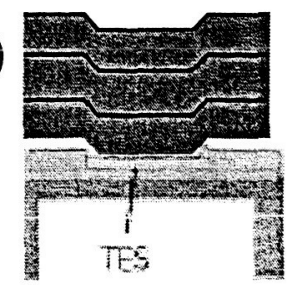

(c)

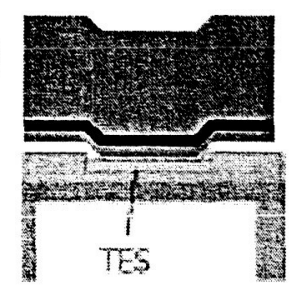

Figure 1. Schematic of the absorber. (a) Top view. (b) Side view of old design. (c) Side view of new design. 

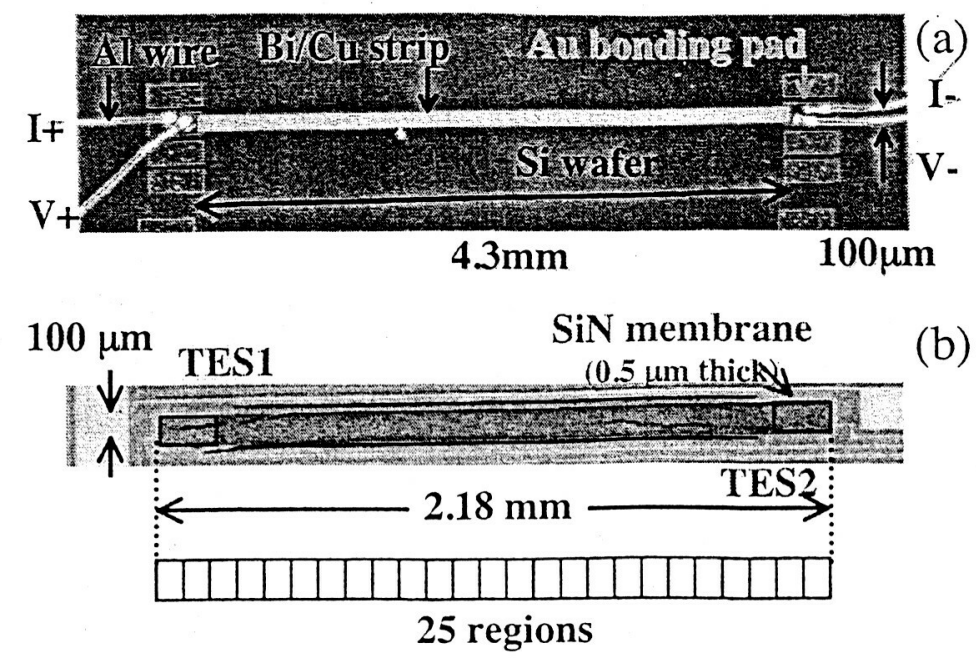

(b)

Figure 2. Pictures of the devices used for (a) the electrical conductivity measurements and (b) for the thermal conductivity measurements.

However, Bi has a low thermal conductivity, which might result in position dependence of the signals from $\mathrm{X}$-rays absorbed within the $250 \mu \mathrm{m} \times 250 \mu \mathrm{m}$ absorber. This would make the energy resolution bad. The optimal filter process, which is a standard process to determine the pulse height of the X-ray microcalorimeter, assumes that all pulses have the same shape. If the pulses have different shapes, the optimal filter gives us wrong pulse heights. Therefore, we put $\mathrm{Cu}$ on the $\mathrm{Bi}$ in order to make the thermal conductivity higher. Since $\mathrm{Cu}$ has a high heat capacity, we need to find the optimal thickness of the $\mathrm{Cu}$ layer. We would like to match the heat capacity to the width of the superconducting transition, so that the dynamic range of the calorimeter covers the whole x-ray band. For our typical transitions, we need to augment the Bi heat capacity anyway. Ideally, the amount of $\mathrm{Cu}$ needed to tune the dynamic range would also provide enough thermal conductivity.

Considering only the X-ray stopping power and the heat capacity, we have been fabricating absorbers with $6.5 \mu \mathrm{m}$ of $\mathrm{Bi}$ and $0.3-1.0 \mu \mathrm{m}$ of $\mathrm{Cu}$ in total. For most absorbers, we have divided the $\mathrm{Bi}$ and $\mathrm{Cu}$ into three layers each, as in Figure 1b. (This multi-layer design was originally implemented to balance stress in thick Bi films.) So far, we cannot reject the possibility that bad thermalization in the absorber degrades the energy resolution. Since the $\mathrm{Cu}$ thickness was chosen based on heat capacity alone, we decided to investigate the electrical and thermal conductivities of our BiCu films, using the devices shown in Figure 2.

\section{THEORIES}

\subsection{Diffusion Equation}

In order to study the non-steady heat conduction, we need to solve the diffusion equation,

$$
\frac{\partial T}{\partial t}=D\left(\frac{\partial^{2}}{\partial x^{2}}+\frac{\partial^{2}}{\partial y^{2}}+\frac{\partial^{2}}{\partial z^{2}}\right) T
$$

where $T$ is the temperature, $t$ is time, $x, y$ and $z$ are position, and

$$
D=\frac{K}{C}
$$

is the diffusion constant. Here, $C$ is the heat capacity per unit volume and $K$ is the thermal conductivity. The propagation time is independent of the cross section but dependent on the square of the distance. 

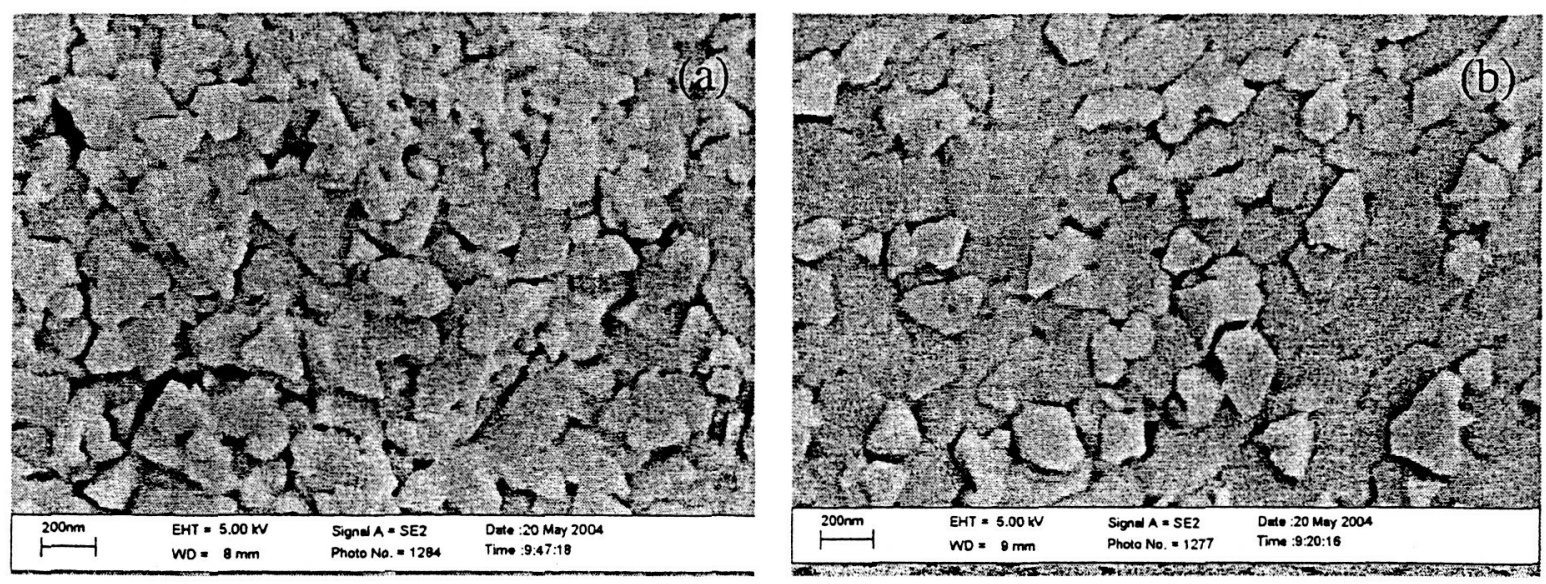

Figure 3. SEM pictures of the Bi strips. (a) $2.25 \mu \mathrm{m}$ thick. (b) $0.25 \mu \mathrm{m}$ thick.

\subsection{Thermal Conductivity}

If we study the steady heat conduction, although that is beyond the scope of this paper, we can measure the thermal conductance of the device, $G$, using the Fourier's law,

$$
G=\frac{Q}{\Delta T}
$$

where $Q$ is the heat current through unit area per unit time and $\Delta T$ is the difference of the temperature of the two thermometers.

\subsection{Wiedemann-Franz Law}

We can estimate the thermal conductivity of the strip from the electrical resistivity, $\sigma$, which we will measure in $\S 4$, using the Wiedemann-Franz law,

$$
K=L T \sigma,
$$

where $L=2.45 \times 10^{-8} \mathrm{~W} \Omega \mathrm{K}^{-2}$ is the Lorentz number.

\section{SEM PICTURES}

We deposited $2.25 \mu \mathrm{m}$ of $\mathrm{Bi}$ on a Si substrate with a deposition rate of $2 \mathrm{~nm} \mathrm{~s}^{-1}$ by a thermal evaporation process without substrate heating. This is our normal deposition method for the $\mathrm{Bi}$, except that we put the $\mathrm{Bi}$ on a TES that is on a SiN membrane. Figure 3a shows a SEM picture of the $2.25 \mu \mathrm{m}$-thick Bi film. The Bi film has rough surface with a grain size of $\sim 0.1 \mu \mathrm{m}$, which is in the same order of the thickness of the $\mathrm{Cu}$ layer. We also deposited $0.25 \mu \mathrm{m}$ of $\mathrm{Bi}$ with a deposition rate of $0.2 \mathrm{~nm} \mathrm{~s}^{-1}$ for comparison. We found that the thinner Bi film has similar grain size, but has smoother surface than the $2.25 \mu \mathrm{m}$-thick Bi film has (Figure $3 \mathrm{~b}$ ).

\section{ELECTRICAL CONDUCTIVITY MEASUREMENTS}

\subsection{Setup}

Figure 2a shows the device used for the electrical conductivity measurements. On a Si substrate, we put $\mathrm{Au}$ bonding pads and deposited a Bi strip on the bonding pads and the Si substrate. On some of the Bi strips, we deposited Cu. The lengih and widith of the strip are $430 \hat{0} \mu \mathrm{m}$ and $100 \hat{0} \mu$, respectively.

We measured the resistance of the strips as a function of the temperature down to $4 \mathrm{~K}$ by 4 -wire measurements, because such measurements enable a quick and comprehensive survey of the $\mathrm{BiCu}$ films. 

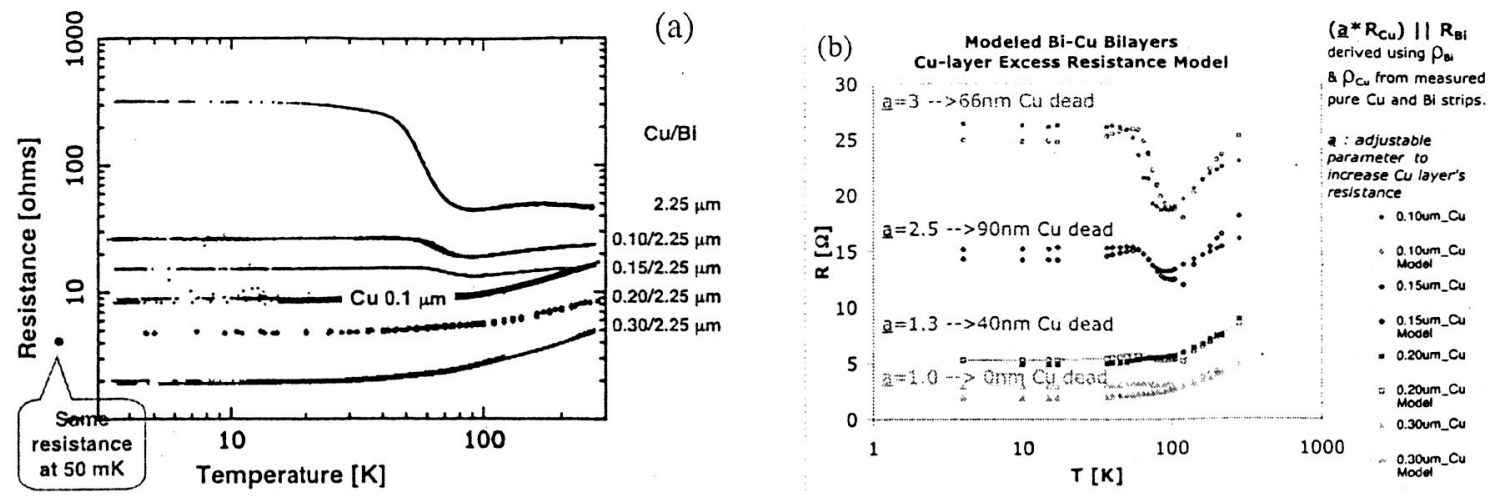

Figure 4. (a) Resistance vs. temperature of the $\mathrm{Bi}$ strip, $\mathrm{BiCu}$ strips and $\mathrm{Cu}$ strip. (b) Reproduced resistance vs. temperature curves of the $\mathrm{BiCu}$ films as a parallel sum of those of the pure $\mathrm{Bi}$ film and the pure $\mathrm{Cu}$ film. Filled and Open symbols represent the actual data and reproduced data, respectively.

\subsection{Resistance vs. Temperature}

Figure 4a shows the resistance vs temperature of a pure $\mathrm{Bi}$ strip, four $\mathrm{BiCu}$ strips, and a pure $\mathrm{Cu}$ strip.

In both of the thin $(0.25 \mu \mathrm{m})$ and the thick $(2.25 \mu \mathrm{m})$ Bi strips, the resistivity increases at low temperatures, and their resistivities are similar to each other. In the literature, $\mathrm{Bi}$ films also show similar temperature dependence of the resistance, although our $\mathrm{Bi}$ film has relatively high resistivity in comparison with other $\mathrm{Bi}$ films (e.g. Ref.2, Ref.3 and Ref.4) suggesting a shorter mean-free path of the electron.

On the $2.25 \mu \mathrm{m}$-thick Bi strips, we deposited $0.1 \mu \mathrm{m}, 0.15 \mu \mathrm{m}, 0.2 \mu \mathrm{m}$ and $0.3 \mu \mathrm{m}$ of $\mathrm{Cu}$, respectively. At low temperatures, since the $\mathrm{Bi}$ has high resistivity, the conductivity in the whole strip is dominated by the $\mathrm{Cu}$, even in the strip with the thinest $\mathrm{Cu}$ layer $(0.1 \mu \mathrm{m})$, while at higher temperatures, the Bi layer makes some contribution to the conductivity of the whole strip when the Cu layer is thin $(0.1 \mu \mathrm{m}$ and $0.15 \mu \mathrm{m})$. Consequently, the $\mathrm{BiCu}$ strip with the thin $\mathrm{Cu}$ layer shows the temperature dependence similar to the pure $\mathrm{Bi}$ strips. At $50 \mathrm{mK}$, we measured the resistance of a $\mathrm{BiCu}$ strip with $0.2 \mu \mathrm{m}$ of $\mathrm{Cu}$ and found that the strip has the same resistances at $50 \mathrm{mK}$ and $4 \mathrm{~K}$. This result allows us to assume that other $\mathrm{BiCu}$ films also have the same resistances from $50 \mathrm{mK}$ through $4 \mathrm{~K}$. We use this assumption when we estimate the thermal conductivity from the electrical conductivity in $\S 4.5$.

We measured the resistance of a $0.1 \mu$ m-thick pure $\mathrm{Cu}$ strip, for comparison with $\mathrm{Cu}$ on $\mathrm{Bi}$. Interestingly, the strip shows three times lower resistance than that of the $\mathrm{BiCu}$ strip with $0.1 \mu \mathrm{m}$ of $\mathrm{Cu}$.

\subsection{Effective thickness of the $\mathrm{Cu}$ layer}

In order to explain the discrepancy in the conductivity for the same $\mathrm{Cu}$ thickness, seen in $\S 4.2$, we tried to reproduce the resistance vs temperature curves of the $\mathrm{BiCu}$ strips assuming that the resistance of the $\mathrm{BiCu}$ strip is a parallel sum of the resistances of the $\mathrm{Bi}$ layer and the $\mathrm{Cu}$ layer. We found that we can reproduce the $\mathrm{BiCu}$ strip with $0.1 \mu \mathrm{m}, 0.15 \mu \mathrm{m}, 0.2 \mu \mathrm{m}$ and $0.3 \mu \mathrm{m}$ of $\mathrm{Cu}$ as a parallel sum of the $2.25 \mu \mathrm{m}$ of $\mathrm{Bi}$ and 0.03 $\mu \mathrm{m}, 0.06 \mu \mathrm{m}, 0.16 \mu \mathrm{m}$ and $0.3 \mu \mathrm{m}$ of $\mathrm{Cu}$, respectively, as shown in Figure $4 \mathrm{~b}$. The results indicate that the $\mathrm{Cu}$ layer is more resistive on a $\mathrm{Bi}$ layer than on a $\mathrm{Si}$ substrate, as if the effective thickness of the $\mathrm{Cu}$ layer were thinner on the $\mathrm{Bi}$ layer, and that the effect is more significant when the $\mathrm{Cu}$ layer is thinner.

From these results, together with the SEM picture, we can conclude that the rough surface of the Bi film makes the $\mathrm{Cu}$ layer more resistive than expected, and that if the $\mathrm{Cu}$ layer were thick enough to fill in the rough $\mathrm{Bi}$ surface and extend as a continuous layer above the $\mathrm{Bi}$, the strip would have the conductivity expected from the pure $\mathrm{Cu}$ result. The SEM pictures suggest that the effective thickness of a $\mathrm{Cu}$ layer could be thicker on a thinner Bi film. In the future, we will fabricate such strips to test this expectation. Considering these results, we designed a new absorber as shown in Figure 1c. We put $1.0 \mu \mathrm{m}$ of Bi on the TES as an insulator, and 0.5 $\mu \mathrm{m}$ of $\mathrm{Cu}$ on the $\mathrm{Bi}$ as a heat bus. We expect that the thin $\mathrm{Bi}$ layer and the thick $\mathrm{Cu}$ layer make the effective 
thickness of the $\mathrm{Cu}$ layer similar to the deposited thickness. On the $\mathrm{Cu}$ layer, we put $5.5 \mu \mathrm{m}$ of $\mathrm{Bi}$ as the $\mathrm{X}$-ray absorber.

\subsection{Annealing}

It is known that we can improve the electrical conductivity of the $\mathrm{Bi}$ and $\mathrm{Cu}$ by annealing (e.g. Ref. 4). Therefore, we annealed the strips 2 hours at $150 \mathrm{C}$ in vacuum.

The resistance vs temperature of the Bi strip didn't change after the annealing. We attempted annealing at higher temperatures on a hot plate. However, the Au bonding pads changed color with 15 minutes annealing at $200 \mathrm{C}$ and melted with 30 minutes annealing at $230 \mathrm{C}$, so that we cannot measure the resistance of the annealed strips with the $\mathrm{Au}$ bonding pads. We are fabricating $\mathrm{Bi}$ strips with $\mathrm{Cu}$ bonding pads to try the annealing at higher temperatures. Note that, however even if we can improve the conductivity, we cannot apply the annealing to the absorber that is deposited directly on the Au of the TES. We need to modify the fabrication process in order to anneal the absorber on the TES at $200 \mathrm{C}$ or higher.

The electrical conductivity of the pure $\mathrm{Cu}$ strip improved, but the $\mathrm{BiCu}$ strips became more resistive due to the annealing. Possible explanations of this are inter-diffusion of the $\mathrm{Cu}$ into the $\mathrm{Bi}$ or $\mathrm{Bi} \mathrm{Cu}$ alloying, although the temperature for the $\mathrm{Bi} \mathrm{Cu}$ alloying is higher than $150 \mathrm{C}$. The $\mathrm{BiAu}$ strips changed color from gold to silver by the annealing, also indicating inter-diffusion or alloying. The rough surface of the Bi supports an inter-diffusion hypothesis.

\subsection{Estimation of the Thermal Conductances}

We estimated the thermal conductivity of the $\mathrm{Bi}$ strip and $\mathrm{Cu}$ strip from the electrical conductivity using the Wiedemann-Franz law: $K$ of the $\mathrm{Bi}$ is $2.0 \times 10^{-10} \mathrm{~W} \mathrm{~K}^{-1} \mu \mathrm{m}^{-1}$ and $K$ of the $\mathrm{Cu}$ is $1.6 \times 10^{-7} \mathrm{~W} \mathrm{~K}^{-1} \mu \mathrm{m}^{-1}$. $K$ of the $\mathrm{Bi}$ is three order of the magnitude higher than that of the $\mathrm{Bi}$. It is known from the literature that $C$ of the $\mathrm{Bi}$ is $1.5 \times 10^{-7} \mathrm{~J} \mathrm{~K}^{-1} \mathrm{~cm}^{-3}$ and $C$ of the $\mathrm{Cu}$ is $1.2 \times 10^{-5} \mathrm{~J} \mathrm{~K}^{-1} \mathrm{~cm}^{-3}: C$ of the $\mathrm{Cu}$ is two orders of magnitude higher than that of the $\mathrm{Bi}$. Using the $C$ values, we can estimate the diffusion constant of the strips as $D$ of the $\mathrm{Bi}$ is $1.3 \times 10^{3} \mu \mathrm{m}^{2} \mu \mathrm{s}^{-1}$ and $D$ of the $\mathrm{Cu}$ is $1.4 \times 10^{4} \mu \mathrm{m}^{2} \mu \mathrm{s}^{-1}$. Cu has $D$ that is one order of magnitude higher than that of the Bi.

In the horizontal directions, the absorber consists of the $\mathrm{Bi}$ layers and $\mathrm{Cu}$ layers in parallel, so that we use the average $K$ and $C$ of the $\mathrm{Bi}$ and $\mathrm{Cu}$ in the diffusion equation. If the $\mathrm{Cu}$ layer is thick enough to dominate $C$ and $K$ of the whole device, $D$ of the whole device approaches $D$ of pure $\mathrm{Cu}$. In other words, there is an upper limit on $D$; if we use too much $\mathrm{Cu}$, it does not further improve the thermalization.

In the vertical direction, we have $6.5 \mu \mathrm{m}$ of $\mathrm{Bi}$ in total without any parallel layer of the $\mathrm{Cu}$. So far, we have assumed that we can ignore thermal transport delay in the vertical direction, but we will assess this assumption with measurements on pure $\mathrm{Bi}$ in the future.

For the $\mathrm{BiCu}$ films, we converted the thermal conductivity into the thermal conductance as

$$
G=\frac{A}{d} K=L T \frac{1}{R}
$$

where $A$ and $d$ are the cross-section and length of the strip, respectively. Here, we used $T=125 \mathrm{mK}$, and $d=$ $2180 \mu \mathrm{m}$, which is the operating temperature and the length of the device used for the thermal conductivity measurement in $\S 5$, because we will compare the estimated $G$ to the result of the thermal conductivity measurement. Filled circles in Figure $4 \mathrm{~b}$ show the estimated $G$ as a function of the Cu thickness. We also show the result from the pure $\mathrm{Bi}$ layer in the figure, for comparison.

So far, we implicitly assumed that the thermal conductance is proportional to the electrical conductance. However we note that it is difficult to apply the Wiedemann-Franz law at such a low temperatures. It is known that $L$ is much smaller than the value shown above at low temperatures (Ref. 5), so that the estimated thermal conductivities are over-estimated. We need the thermal conductivity measurements in order to confirm the assumptions. 


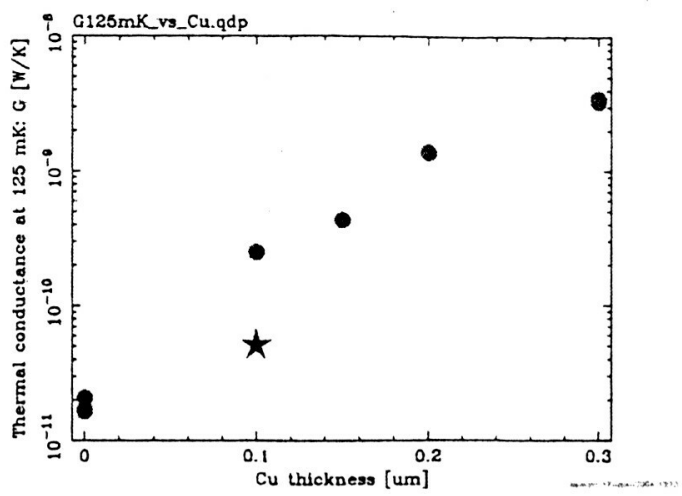

Figure 5. Filled circles show the thermal conductance of the Bi strip and the BiCu strips at $125 \mathrm{mK}$, estimated from the electrical resistivity. A filled star shows measured thermal conductance of the BiCu strip with $0.1 \mu \mathrm{m}$ of $\mathrm{Cu}$.

\section{THERMAL CONDUCTIVITY MEASUREMENT}

\subsection{Setup}

\subsubsection{Devices}

Figure $2 \mathrm{~b}$ shows the device used for the thermal conductivity measurements. Each device has two TESs and a long absorber on a SiN membrane. The membrane has slits along the absorber, so that all the heat in the absorber should flow through the two TESs. This device is a kind of Position Sensitive TES microcalorimeter (PoST; see Sadleir et al. and Figueroa-Feliciano et al. in this Volume) with a continuous absorber, so that we can determine the X-ray hit position without any collimator. The ratio of the pulse heights of the two TESs tells us the position.

The membrane has a thickness of $0.5 \mu \mathrm{m}$. Each TES has a transition temperature of $\sim 127 \mathrm{mK}$. On the membrane and the TESs, we deposited $2.25 \mu \mathrm{m}$ of $\mathrm{Bi}$ and $0.1 \mu \mathrm{m}$ of $\mathrm{Cu}$. The length of the absorber is 2180 $\mu \mathrm{m}$, of which $190 \mu \mathrm{m}$ overlaps each of the two TESs. The width of the absorber is $100 \mu \mathrm{m}$.

\subsubsection{Measurements}

We put the device into an ADR and cooled down the heat bath to $120 \mathrm{mK}$. We applied bias voltages to the two TESs in the PoST. We tuned each bias voltages to obtain similar pulses from the TESs. We irradiated the device with characteristic X-rays from $\mathrm{Ti}$. We read out signals from the two TESs using two two-stage SQUIDs with a sampling rate of $1 \mathrm{MHz}$ and a cutoff frequency of $300 \mathrm{kHz}$. We utilized a digital oscilloscope to collect the raw data. In order to investigate performance of the devices, we analyzed the raw pulse shapes without using an optimal digital filter.

\subsubsection{Event Selection}

We measured the pulse height of each X-ray event as shown in Figure 6a. We see two distributions corresponding to the Ti-K $\alpha$ and $\beta$ lines, although it is not easy to separate them at the ends of the distribution. We also measured the area of the pulse for each X-ray event, by integrating the pulse after making the DC level zero. We plot the area of the pulse of TES2 against that of TES1 in Figure 6b. We see a clear separation between $\mathrm{Ti}-\mathrm{K} \alpha$ and $\mathrm{Ti}-\mathrm{K} \beta$ lines. We selected the events in the $\mathrm{Ti}-\mathrm{K} \alpha$ line on the area vs area plot for the following analysis.

We divided the $\mathrm{X}$-ray events of the $\mathrm{Ti}-\mathrm{K} \alpha$ line into 25 groups, after sorting the events by the area ratio. We assumed that each region corresponds to one of the 25 regions of the absorber shown in Figure $2 \mathrm{~b}$. We calculated the average pulse for each region as shown in Figure 7. 




Figure 6. (a) Distribution of the pulse height of the two TESs. (b) Distribution of the pulse area of the two TESs.

\subsubsection{Monte-Carlo method}

In order to estimate the diffusion constant of the device, we compared the measured result to results of simulations. We performed a Monte-Carlo method to solve the diffusion equation, assuming diffusion constant of $10^{2}, 10^{3}$ and $10^{4} \mu \mathrm{m}^{2} \mu \mathrm{s}^{-1}$. We assumed a $10 \mu \mathrm{m} \times 10 \mu \mathrm{m}$ mesh. We considered only the horizontal diffusion, because the thickness of the film is negligible.

\subsection{Diffusion Constant}

\subsubsection{Position Dependences}

We measured the pulse height, the pulse area, and the rise time of the 25 average pulscs as a function of position as shown in Figure 8. We see a saturation of the pulse height in Figure 8a. This is because the pulse was not small enough to keep the TES resistance on the transition edge, due to too little heat capacity. We need the low heat capacity for the good energy resolution, but if it is too low, we suffer from the saturation of the pulses. We also see the saturation of the pulse area in Figure 8b, but the saturation is less significant. This is the reason why we used the pulse area ratio instead of the pulse height ratio to determine the position.

We superposed the Monte-Carlo results on Figure 8 as open circles, open squares, and open triangles. We found that diffusion constant of $2 \times 10^{3} \mu \mathrm{m}^{2} \mu \mathrm{s}^{-1}$ reproduces the measured data well.

\subsubsection{Delay of rise}

Figure 9a shows average pulses of the TES1 and TES2 when X-rays hit the absorber on the TES1. The pulses of the TES1 and TES2 were collected by the oscilloscope at the same time. We see a delay of the rise in the TES2 pulse. This means that it takes $\sim 100 \mu \mathrm{s}$ for the heat to travel $2180 \mu \mathrm{m}$. Figure 9b shows the Monte-Carlo pulses of the TES1 and TES2 for the same X-ray hit position that generated assuming a diffusion constant of $10^{3} \mu \mathrm{m}^{2} \mu \mathrm{s}^{-1}$, whose delay of rise is in good agreement with the actual data.

\subsubsection{Theoretical value}

We estimated the diffusion constant assuming the free-electron approximation,

$$
D=\frac{1}{3} v_{\mathrm{f}} l
$$

where $v_{\mathrm{f}}$ is the Fermi velocity and $l$ is the mean free path. If we assume the inferred effective thickness of the $\mathrm{Cu}, 0.03 \mu \mathrm{m}$, as the mean free path, we obtain $1.6 \times 10^{4} \mu \mathrm{m}^{2} \mu \mathrm{s}^{-1}$. This is one order of magnitude larger than the actual diffusion constant. This means that we cannot apply the free-electron approximation at such a low temperature, so we need the actual measurement of the thermal conductivity to estimate the diffusion constant. 


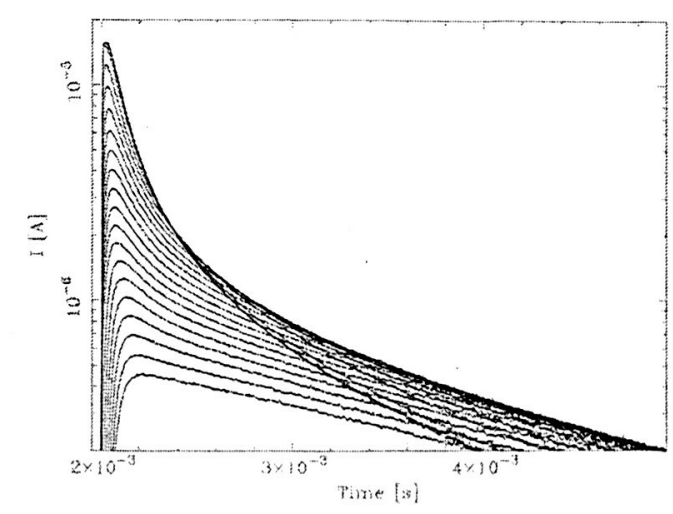

Figure 7. Average pulses of TES1. Each pulse corresponds to one of the $25 \mathrm{X}$-ray hit positions.

\subsubsection{Comparison with the electrical conductivity results}

In Figure 4b, we superposed the actual $G$ determined from the thermal conductivity measurement as a filled star. We converted the diffusion constant into the thermal conductivity using Eq. (2). If we consider the overestimation in the Wiedemann-Franz results, the results of the electrical and thermal conductivity measurements are consistent with each other.

\subsection{Energy Resolution}

We estimated the degradation of the energy resolution due to the position dependence.

\subsubsection{Energy loss}

In the PoST, the X-ray energy is divided into the two TESs, so that the pulse area has the position dependence. However, this does not mean that the pulse area is dependent on the X-ray hit position in the standard TES calorimeter. In the area vs area plot, the events are on straight lines. Since the area of the calorimeter pulse is proportional to the X-ray energy, this means that there is no energy loss, namely all the X-ray energy is collected by the two TESs. In the standard TES calorimeter, there is only one TES, so that all X-ray energy is collected by the one TES, namely the pulse area should be independent of the X-ray hit position.

\subsubsection{Pulse shape}

In Figure 10, we show average pulses of two adjacent regions, after correcting the pulse scale to give equal area, considering the result in $\S 5.3 .1$. The difference of the pulse heights in the two regions is $\sim 20 \%$ of the pulse heights. This means the thermal diffusion constant of the film is too low to get the good energy resolution. Fortunately, $0.1 \mu \mathrm{m}$ of $\mathrm{Cu}$ is much thinner than the amount needed to meet the design heat capacity in the 250 $\mu \mathrm{m}$ wide pixels, for typical transition widths and using published values for heat capacity in $\mathrm{Bi}$ and $\mathrm{Cu}$.

There is a possibility that our Bi film has a much higher heat capacity than expected, so that the $0.1 \mu \mathrm{m}$ $\mathrm{Cu}$ layer is not thick enough to dominate the heat capacitance in the whole absorber. We can determine the thermal conductivity of the SiN membrane by the IV measurements, namely by applying a bias voltage to the device and measuring the current through the TES. We can roughly determine the ratio between the heat capacity of the device and the thermal conductance of the SiN membrane using the decay time of the pulse. Consequently, we can determine the heat capacitance of the device using the IV curves and the decay time of the pulse. In the present case, we obtained a heat capacitance that is 1-2 orders of magnitude higher than the expected value. We need to understand the $\mathrm{Bi}$ contribution to the heat capacity before we can determine the optimal $\mathrm{Cu}$ thickness. 

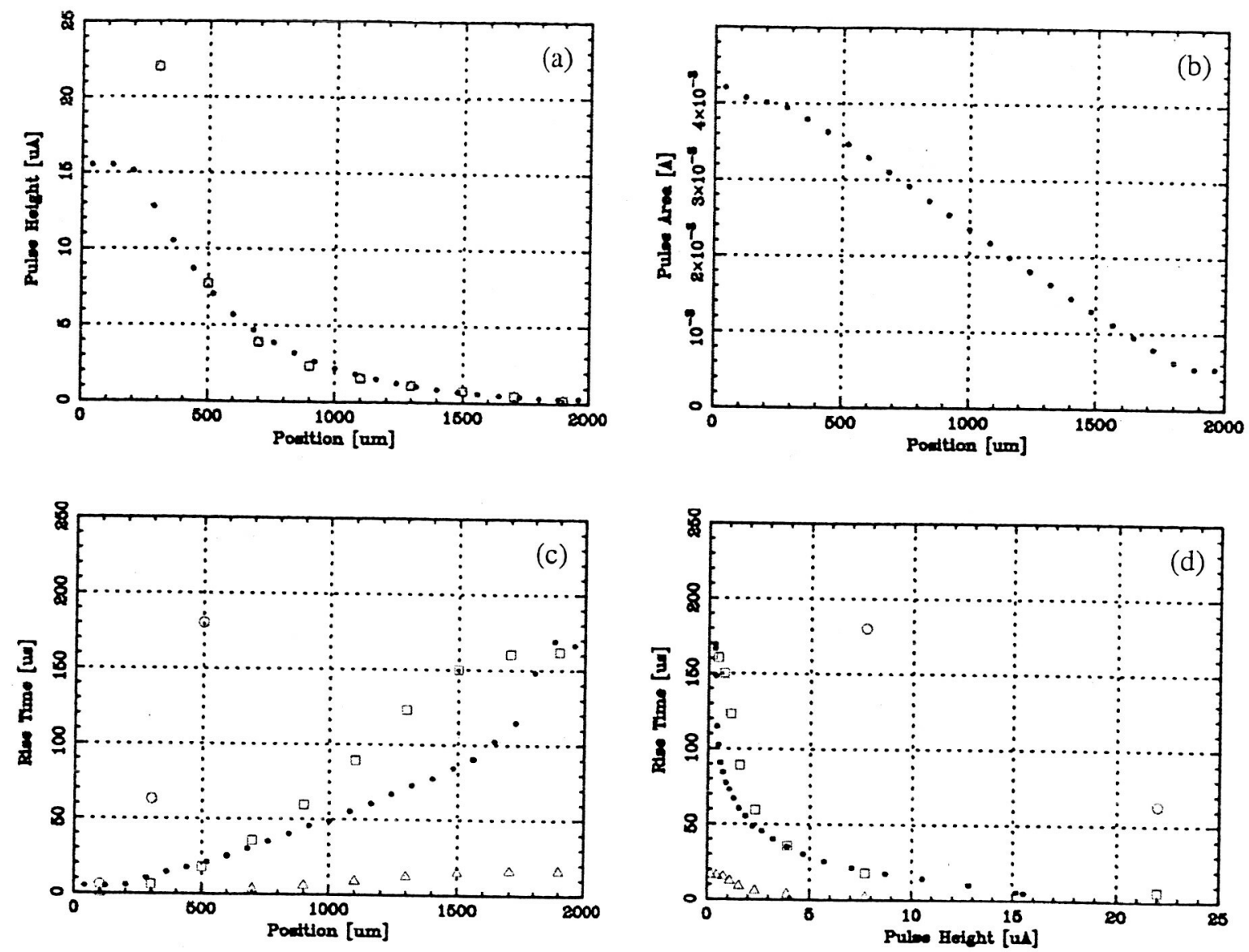

Figure 8. Position dependences of (a) the pulse height, (b) the pulse area and (c) the rise time. (d) Rise time vs pulse height. Filled circles represent the actual data. Open circles, open squares and open triangles represent the simulated data with a diffusion constant of $10^{2}, 10^{3}$ and $10^{4} \mu \mathrm{m}^{2} \mu \mathrm{s}^{-1}$, respectively.
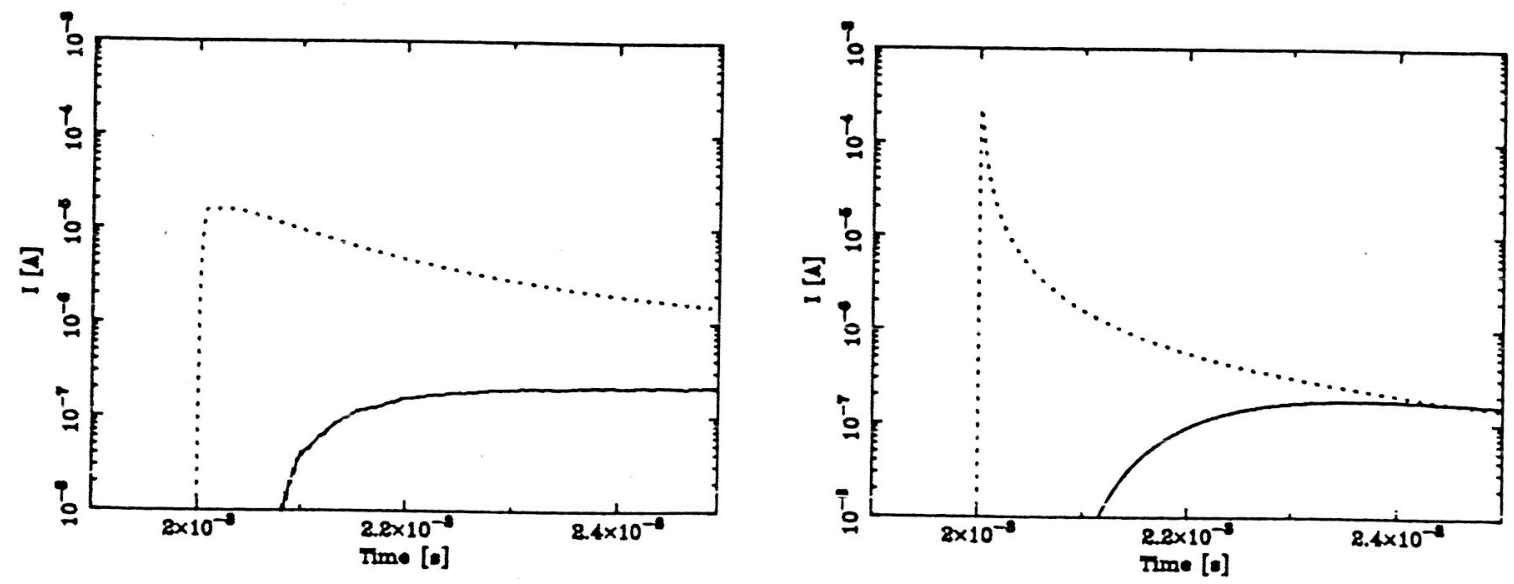

Figure 9. Comparison of the TES1 (dotted line) and TES2 (solid line) pulses when an X-ray hits the absorber on the TES1. (a): actual pulses. (b): simulated pulses. 


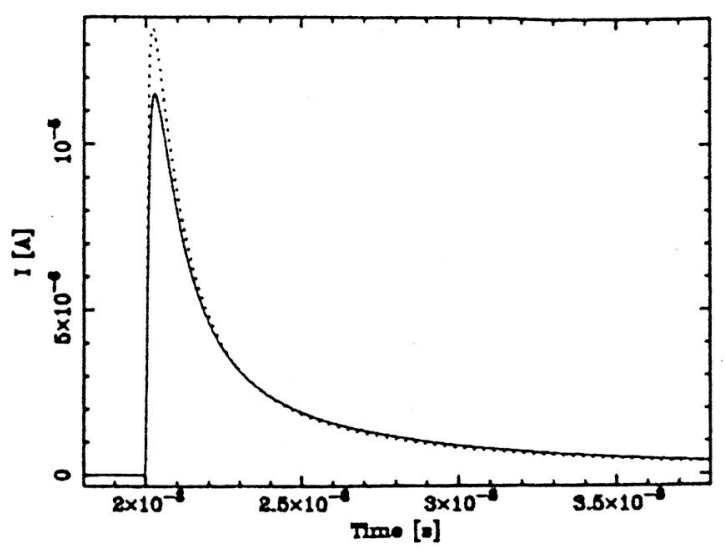

Figure 10. Comparison of the pulse shape of the 20th (solid line) and 21st (dot line) regions of the 25 regions, shown after the area correction.

\section{FUTURE PLANS}

\subsection{Improvement of the Bi films}

We will try to improve our Bi film, especially the smoothness of the Bi surface, by annealing after the deposition, by heating the substrate during the deposition, and/or by slow-rate deposition. We will test these devices by taking the SEM pictures and by measuring the resistance versus temperature.

\subsection{Electrical Conductivity Measurements}

We will also deposit $\mathrm{Cu}$ on the thinner $\mathrm{Bi}$ and measure its electrical resistance to confirm the expectation that the rough surface of the $\mathrm{Bi}$ film makes the $\mathrm{Cu}$ resistive.

\subsection{Thermal Conductivity Measurements}

We will fabricate and test a device with pure Bi to measure the thermal conductivity of the Bi film itself and examine whether the vertical thermal resistance in the $\mathrm{Bi}$ is negligible or not. If it is not negligible, we need to improve the design of the absorber, for example by adding a vertical $\mathrm{Cu}$ layer for the thermal conductivity.

We will fabricate and test the thermal conductivity measurement devices with thinner $\mathrm{Bi}$ and/or thicker $\mathrm{Cu}$. If the resistivity of the $\mathrm{Cu}$ is improved thanks to the use of a single thicker $\mathrm{Cu}$ layer and the smoother surface of the thin $\mathrm{Bi}$, the thermal conductivity of the $\mathrm{Cu}$ increases but the heat capacity of the $\mathrm{Cu}$ stays the previous value, so that we can get a larger diffusion constant. We will also fabricate more thicker $\mathrm{Cu}$ devices to test whether the diffusion constant becomes higher or not.

In this paper, we investigated the time-dependent heat conduction using the X-ray pulse as a heat source and measured the diffusion constant. Added to this, if we can study the steady heat conduction to measure the thermal conductivity directly, we can determine $C$ of our Bi film, which is suspected to be much higher than the normal value, by dividing the diffusion constant by the thermal conductivity. We can use the two TESs of the device as the thermometers for the thermal conductivity measurements and can also use one of them as a heater to supply the heat current.

\section{REFERENCES}

1. C. K. Stahle proc SPIE 3765 , p. 82, 1999.

2. R. A. Hoffman and D. R. Frankl Physical Review B 3, p. 1825, 1971.

3. D. E. Beutler and N. Giordano Physical Review B 38, p. 8, 1988.

4. Y. Kim Applied Physics Letters 79, p. 3651, 2001.

5. K. C., Introduction to Solid State Physics, John Wiley \& Sons, Inc., New York, 1986. 